\title{
A VIRTUAL LABORATORY FOR AN ENHANCED AND SAFE UNDERSTANDING OF THE ELECTRIC TRANSFORMERS OPERATION
}

\author{
Jorge Bonet-Jara ${ }^{1}$, Joan Pons-Llinares ${ }^{2}$, Soledad Bernal Pérez ${ }^{1}$, Roser Sabater I \\ Serra ${ }^{1}$ \\ ${ }^{1}$ Departamento de Ingeniería Eléctrica, Universitat Politècnica de València (SPAIN) \\ ${ }^{2}$ Instituto de Ingeniería Energética, Universitat Politècnica de València (SPAIN)
}

\begin{abstract}
The paper presents an educational software designed to simulate the behaviour of an electric transformer under no-load, shortcircuit and load test, measuring its input and output magnitudes through network analysers, and enabling the supply voltage and load regulations. This virtual laboratory allows to achieve several objectives. First, the student becomes familiar with the system to be operated in the real laboratory, reducing the subsequent electrical risk and optimizing the time required for carrying out the practice. Second, it enhances the understanding of transformers behaviour, its modelling through the equivalent electrical circuit, and the calculation of its two most characteristic parameters during its operation (performance and internal voltage drop). Finally, the application auto evaluates the transformer characterization performed by the student.
\end{abstract}

The simulator reproduces schematically, but precisely, the assembly that the student faces during the lab lesson: an autotransformer to regulate the supply voltage, a transformer, two input and output network analysers, and different types of loads. The tool is structured in three panels. In the first panel, the student selects a transformer from 24 options, and performs its shortcircuit and no-load tests to characterize it through its electric equivalent circuit. Panels 2 and 3 enable load tests and its main characteristics calculation (performance and internal voltage drop). The student results are introduced in the app to perform auto-evaluation.

To measure the performance and usefulness of the application, it has been implemented in the lab lessons of the subject "Electrical Technology", belonging to the Bachelor's Degree in Industrial Electronics and Automation Engineering, taught at the Polytechnic University of Valencia (Spain). The practice lessons follow this sequence: a refreshment of the transformer concepts needed + explanation of the practice lesson to be performed + explanation and use of the app solving an example + real laboratory experience + final test on transformers. The sample size is 110 students, divided into five groups. The last group does not use the app, and their test results are compared with the four remaining groups, measuring how the app enhances learning. Finally, the last group receives the app explanation, solves an example and repeats the test, showing how their learning is improved.

It is observed that the students obtain better results after using the virtual lab, reaching higher levels of learning. On the other hand, it is also observed that the time of completion of the real laboratory practice decreases substantially. As a conclusion, there is a great benefit in using this application specifically designed to obtain very specific learning results, since the application is fully adapted to the needs of the degree and therefore to the level of knowledge that the students need to acquire according to the perception and experience of the teaching staff. Finally, based on the results, the application is permanently implemented in the laboratory lessons of Electrical Technology.

Keywords: virtual laboratories, e-learning, educational software.

\section{INTRODUCTION}

It has been demonstrated over the last decades that software and educational applications have the virtue of enhancing, increasing and intensifying the degree of learning that a person experiences in a certain area of knowledge, as Kozma maintains in [1]. In this line of work, several articles have been published demonstrating that applying new educational techniques based on the use of computerassisted learning, students obtain better results than those taught by traditional techniques (Smit et al [2], Cingi [3]). 
The advantages of these new learning techniques are even more visible when we focus on the field of engineering, where often the complicated systems and equations that are handled hinder a global vision of the problem and an "on time" resolution. In this regard, many authors emphasize the importance of having computer programs in the classrooms that allow training and instruction of students in various engineering problems: these teaching methods should be extended throughout the universities, as Şeker maintains in [4]. The base of this training and instruction mostly resides in the safe and self-taught formation that the student gets when he is able to, in a fast and simple way, change and play with the diverse parameters of the inputs of processes and systems, which are sometimes complicated, being also able to visualize what effects these modifications have on the output variables. This would not be possible without the help of simulation programs or virtual laboratories. Through these methods, a deeper understanding of the physical principles underlying these problems is achieved, as well as a greater development of procedural abilities and skills when facing those same situations in real life, as demonstrated by Kollöffel \& De Jong [5], with their virtual laboratory for the simulation of processes, systems and phenomena related to electrical circuits.

This paper presents an educational software, in a completely independent executable .exe format, that allows to simulate the operation of a three-phase power transformer (TPPT), to serve the students as help and support in their understanding of the TPPT use and operation. Among all the industrial devices that are taught throughout the course ("Electrical Technology" of the "Degree in Industrial Electronics and Automation Engineering " taught at Universitat Politècnica de València), the TPPT has been chosen because of its high use in industry and its high risk of use in the laboratory. Over the years, it has been observed that the students find serious difficulties when facing the assembly of the TPPT test bench, as well as in the performance of the tests owing to the lack of previous experience. Moreover, the dangerous voltage in these sessions does not allow to quickly perform several tests and increase learning. Therefore, an application that faithfully reproduces the real assembly that students have during that session has been designed and build. The virtual laboratory introduces the student to the TPPT, favouring a fast, safe and effective learning. Moreover, it facilitates the lab tests performed afterwards. To achieve this goal, both the behaviour of a TPPT under its most characteristic tests and the measure and regulation devices are simulated. As far as the authors know, there is currently no application specifically aimed at this purpose and under the proposed format: although it is true that in universities, computer-aided training is frequently used through programs such as Matlab and its extension Simulink, LabView, etc., these require previous knowledge of programming in these languages. However, the application here presented does not require such knowledge, since it has a comfortable graphical user interface: their only concern is interacting with the input and output parameters. To measure the performance of the application, three parameters (student satisfaction, improvement of knowledge level and time) will be analysed throughout test questions, surveys and direct experience from the professors.

\section{OBJECTIVES}

Learning in a high risk laboratory is limited, because it is not possible for the student to easily test the material of the lab due to the electrical danger. Therefore, limited number of tests can be performed in one lab session, restricting the learning. In addition, the lack of students experience with the devices used leads to requiring too often the teacher's help to solve problems that would not exist if they have had, in somehow, previous experience with the devices they are dealing with. That same lack of previous experience with the apparatus of the laboratory also translates into an insecure operation, which can be extremely dangerous, especially considering that they are electrical appliances operating at dangerous voltages. Moreover, an improper devices handling can lead to its damage, falling into, not only more delays, but also to the device destruction.

Due to these two problems, this lab sessions are not only dangerous, but also there is not enough time to reach all the learning objectives. The key to achieve all the goals of learning lies in the level of prior knowledge that the student is able to acquire in a practical and interactive way before entering the laboratory. Therefore, an application that simulates the operation of the laboratory, quickly reproducing diverse operating conditions, is especially useful for the student to interact and play safely with the different devices and their characteristic parameters, learning the basics about its operation as well as assimilating the basic routines to be carried out during lab session. It can be concluded that the final goal is to provide students with an application that allows them to self-acquire a series of prior knowledge in an easy, quick, save and interactive way. Specifically, as regards the TPPT, these objectives of prior knowledge can be subdivided into two classes: those related to the theoretical framework of the operation of the TPPT and those related to the physical operation of it. 


\subsection{Theoretical learning objectives}

The main objective is the understanding of the TPPT through the model known as "Equivalent Electric Circuit" (EEC). The EEC model is based on the representation through resistors and reactances of the electric circuits of the primary and secondary windings and their consequent coupling. So, in permanent regime, you can have an easily solvable circuit away from the resolution of complicated differential equations, which is very useful for the student when it comes to understanding the basic concepts of the transformer. This circuit, depicted at the bottom of Fig. 1, consists of four parameters:

- $-R_{c c}:$ Shortcircuit resistance.

- $-X_{c c}$ : Shortcircuit reactance.

$-\quad-R_{F e}$ : Iron losses resistance.

- $X_{\mu}$ : Magnetizing reactance.

The objective is that the students understand and are able to obtain (through no-load and shorcircuit tests), the four parameters that characterize the EEC. Moreover, since these tests are easily and quickly performed with the virtual laboratory, which contains data of several TPPTs with different powers, the students can perform several tests and observe how the CEE parameters vary with the power of the TPPT chosen. This learning goal is impossible to be achieved in a physical laboratory.

As a second objective, the students will be familiarized with the two most characteristic parameters of a TPPT during its load operation: the internal voltage drop and the performance. The virtual laboratory allows the students to see the characteristic curves associated with these two parameters and how they vary according to the level and type of load (more or less inductive/resistive/capactive). Finally, the students obtain the values of these parameters in different operation points simulated (different level and type of load), and the app allows them to depict these values together with the entire curve.

\subsection{Practical learning objectives}

Through the use of the application, the students must learn how to perform the tests (shortcircuit, noload and load), and more particularly what order to follow with the tests, the connections to be made, and the conditions to be set. These three questions are addressed through the application as follows:

The short circuit test is the most dangerous to be performed by the students, which is why it is the first to be carried out and under strict supervision of the teacher. In order to make the student aware of this, the application shows the tests sequentially: shortcircuit, no-load and load.

The graphical interface guides the student through the sequence of connection between the different devices in the test bench, so that when he faces the assembly in the lab, he has a basic idea of what equipment he is going to use and which is the order of connection.

The supply conditions are very different for each test, and the confusion between one test and another can lead to disastrous effects for the TPPT itself and for the different measuring devices coupled. For this reason certain control elements have been placed in the application in order to indicate the student if he has exceeded or has not reached the limit conditions shown in Table 1.

Table 1. Supply conditions.

\begin{tabular}{|c|c|c|c|}
\cline { 2 - 4 } \multicolumn{1}{c|}{} & Shortcircuit & No-load & Load \\
\hline Supply & Rated current & Rated voltage & Rated voltage \\
\hline
\end{tabular}

Therefore, the student will learn with the use of the application the steps to take to perform all the tests and the conditions that must be set in each of them. Moreover, especial emphasis will be placed, though the application, on the softness with which the supply voltage has to be varied during the shorcircuit test in order not to reach levels of currents that could melt on of the windings or make the protections trip. 


\section{METHODOLOGY}

This section describes the methodology applied to measure the impact of the virtual laboratory in the knowledge achieved by the students that take the TPPT laboratory sessions of the subject "Electrical Technology". First of all, the sequence of activities that take place during the laboratory session must be clarified:

- Brief theoretical introduction: the key theoretical concepts dealing with the TPPT, which will be used and embraced during the lab session, are presented.

- Use of the virtual laboratory (explained through 3.1): the students follow a detailed guide (without any explanation to improve their autonomous work capabilities), which introduces them to the virtual laboratory and enables to perform the calculations with at least one TPPT; the application enables a self-assessment.

- Laboratory tests: the students enter the real laboratory and physically perform the TPPT tests.

- Evaluation of the learning achieved: a test is performed to evaluate if the students have achieved the learning goals.

The subject has a total of 110 students, which have been divided into 5 groups of 22 people each. Within the same group the students are grouped in pairs of two. In order to evaluate the virtual laboratory performance, one of the groups was asked to use the application after the real laboratory tests and after solving the final test. This way, the different results between this group and the rest can be compared. The test is described in Subsection 3.2, while the results obtained are presented in subsection 4.1. In addition, all the groups were given a satisfaction survey (described in Subsection 3.3), asking them about different aspects of the application and whether it had helped them or not to achieve the learning objectives. The results of this survey are presented in Subsection 4.2.

\subsection{Description of the application}

It is an application programmed and developed through MATLAB using its GUI editor. Despite of that, the app is a standalone, so it does not require MATLAB to work: it only requires an installer and the .exe file. This way, a powerful computer is not required, due to its low computational load. This gives great versatility to the distribution and installation of the software in the different classrooms assigned to the lab sessions.

As mentioned above, the app simulates a virtual laboratory that reproduces in a schematic but precise way, the assembly that students will later carry out during the lab sessions, so that they can practice with it safely. This assembly consists of: power supply + autotransformer + network analyser in primary winding + transformer + network analyser in secondary winding + load. These elements can be distinguished in centre part of Fig. 1, from left to right, just on top of the EEC drawn. The power supply + autotransformer is simulated through a slider bar that the student can move by means of the arrows at their end for a fine adjustment, or by sliding the bar for a gross adjustment of the voltage value (Fig. 1, left). The network analysers are simulated through two panels at the input and output of the transformer where the values of voltage, current, active power and reactive power are shown, in the same way they are shown by the physical laboratory analyser. Finally, transformer and load are represented schematically (centre and right par of Fig. 1), the first through its rated values, and the second through the active and reactive power that the TPPT would consume if it was ideal.

The entire application is structured in three panels (explained in the three following subsections). The panels are dedicated to totally different activities, but interrelated. The first panel is dedicated to obtaining the four parameters that characterize the EEC, the second to obtain the internal voltage drop and the performance of the transformer by an exact method, and the third to obtain these same two parameters but by an approximate method.

The procedure to follow is: the application is initialized, a transformer is selected from among the 24 available (top-left selector in Fig. 1), the rated values of it are shown in the transformer (represented in the middle), and the different tests and loads are selected (top-right selector in Fig. 1). The activities of the three panels are then completed (the first panel is the one selected by default). A detailed guide has been written so as to allow students to perform a totally autonomous use of the application, favouring a transversal competence such as autonomous learning and culminating in self-evaluation by the applications itself. 


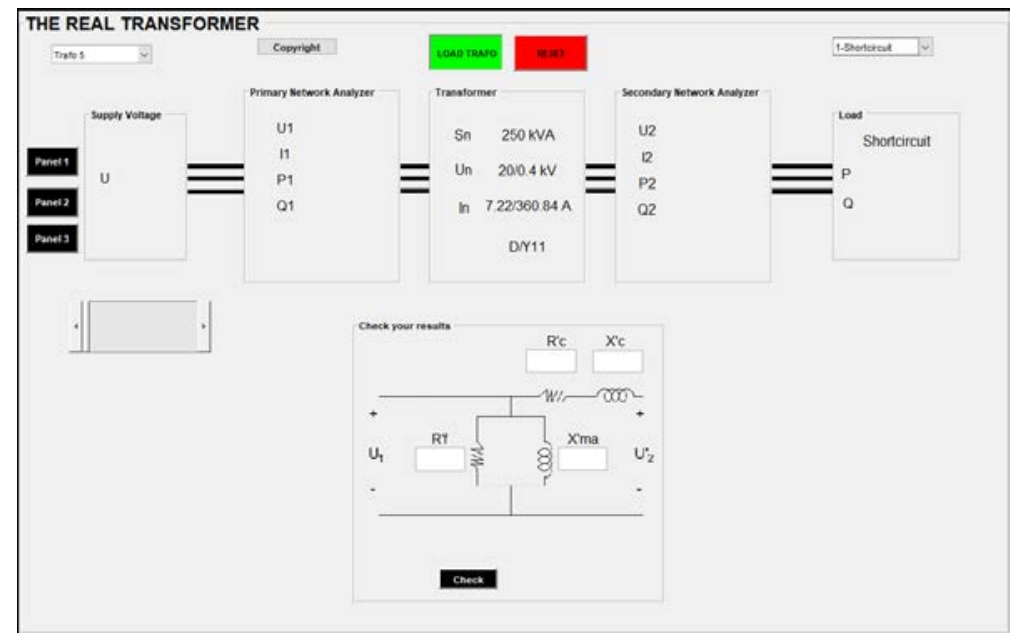

Figure 1. General view before starting the shortcircuit test.

\subsubsection{Panel 1: Obtaining the equivalent circuit parameters.}

The objective of this panel is to provide the students with experience in obtaining the EEC parameters through no-load and short circuit tests. In addition, it is intended that students assimilate the supply conditions associated with these two types of tests to improve their security and that of the devices used when the real laboratory tests are performed.

Once the transformer is selected and loaded, the student will select the shortcircuit test from the test list, since, as during the lab session, this will be the first test to be carried out. The student must move the slider-bar which regulates the voltage smoothly so as not to exceed the rated current. This way, the student will assimilate and practice that during the shortcircuit test, little voltage variations involve large current variations, so that he has to be quite careful. In order to facilitate this process, four bars have been provided with marked limits (rated limits), one for voltage and the other one for current on both sides of the TPPT, measuring those magnitudes at the entry (primary winding) and exit (secondary) of the TPPT. Those bars increase or decrease with the movement of the slider bar, and therefore with the supply voltage. The current bars increase very quickly for the shortcircuit test.

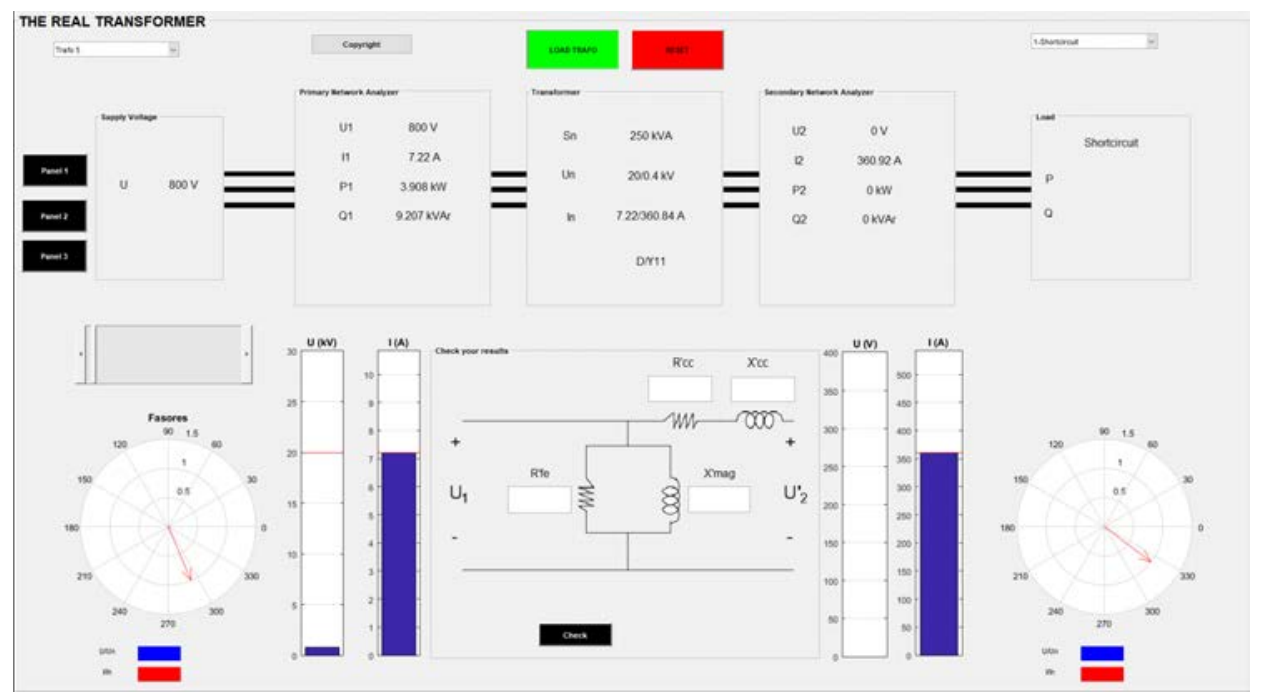

Figure 2. Shortcircuit test.

Finally, the student will use the values of current, active power and reactive power provided by the primary network analyser to obtain the first two parameters of the EEC using the following formulas:

$$
R_{c c}=\frac{P_{1}}{3 I_{1}^{2}} \quad X_{c c}=\frac{Q_{1}}{3 I_{1}^{2}}
$$


Next, the student will select the no-load test to obtain the remaining parameters. In this case, the student will realize that during the no-load test the current flowing through the primary winding is very small so that the voltage regulator can be handled with more ease, varying the voltage until it is equal to the rated value. Once the voltage is adjusted, the student will measure the active power and reactive power and obtain the last two parameters through the following formulas:

$$
R_{F E}=\frac{U_{1}^{2}}{P_{1}} \quad X_{\mu}=\frac{U_{1}^{2}}{Q_{1}}
$$

Finally, the student can verify on the EEC if the results obtained are correct, by entering each of the values in its corresponding box and clicking "Check". A red check will appear in that case:

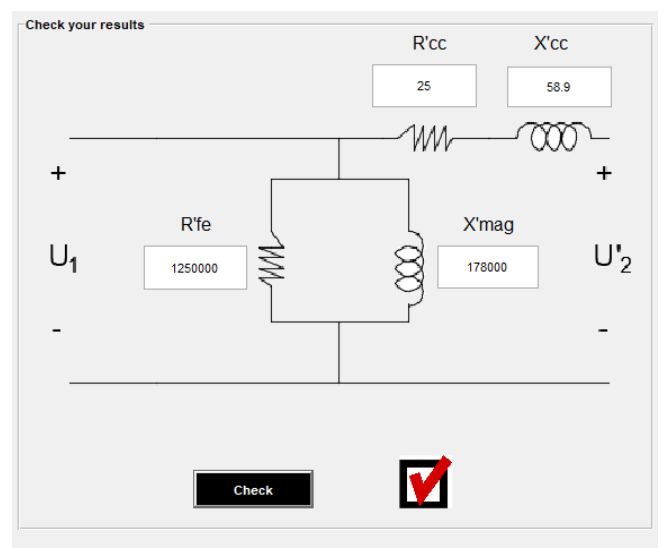

Figure 3. Checking the results of the first activity.

\subsubsection{Panel 2: Internal voltage drop and performance. Exact method.}

This second panel is designed for the student to become familiar with and embrace two of the most important parameters of a transformer operating in load conditions: performance and internal voltage drop. For this purpose, there are nine different loads adapted for each transformer, corresponding to three load levels and three different power factors, with the aim that students sees how the performance and voltage drop are modified according to the typology of the load:

Table 2. Composition of the loads for the load test.

\begin{tabular}{|c|c|c|c|c|}
\hline & & \multirow{2}{*}{\multicolumn{3}{|c|}{ Load level }} \\
\hline & & & & \\
\hline & & 1 & 0.9 & 0.7 \\
\hline \multirow{3}{*}{ Power factor } & 1 & Load 1 & Load 2 & Load 3 \\
\hline & $0.6(\mathrm{i})$ & Load 4 & Load 5 & Load 6 \\
\hline & $0.48(\mathrm{c})$ & Load 7 & Load 8 & Load 9 \\
\hline
\end{tabular}

First, the student will select load number one and bring the voltage level of the primary to its rated value, just as the load test will be carried out during the real lab session. Once the voltage is adjusted, the student will select panel 2 and after recording the values of voltage and active power, he will obtain the internal voltage drop and the performance by means of the following formulas:

$$
\eta=\frac{P_{2}}{P_{1}} \quad \varepsilon_{c}=\frac{\left|U_{2,0}\right|-\left|U_{2}\right|}{\left|U_{2,0}\right|}
$$

Finally, he can check the results by entering the values in each of the boxes show in Fig. 4 and double check them by plotting each result above the performance and voltage drop curves associated with each power factor. In this way, the student will also become familiar with the shapes that these curves usually adopt and how they vary depending on the power factor of the load. 


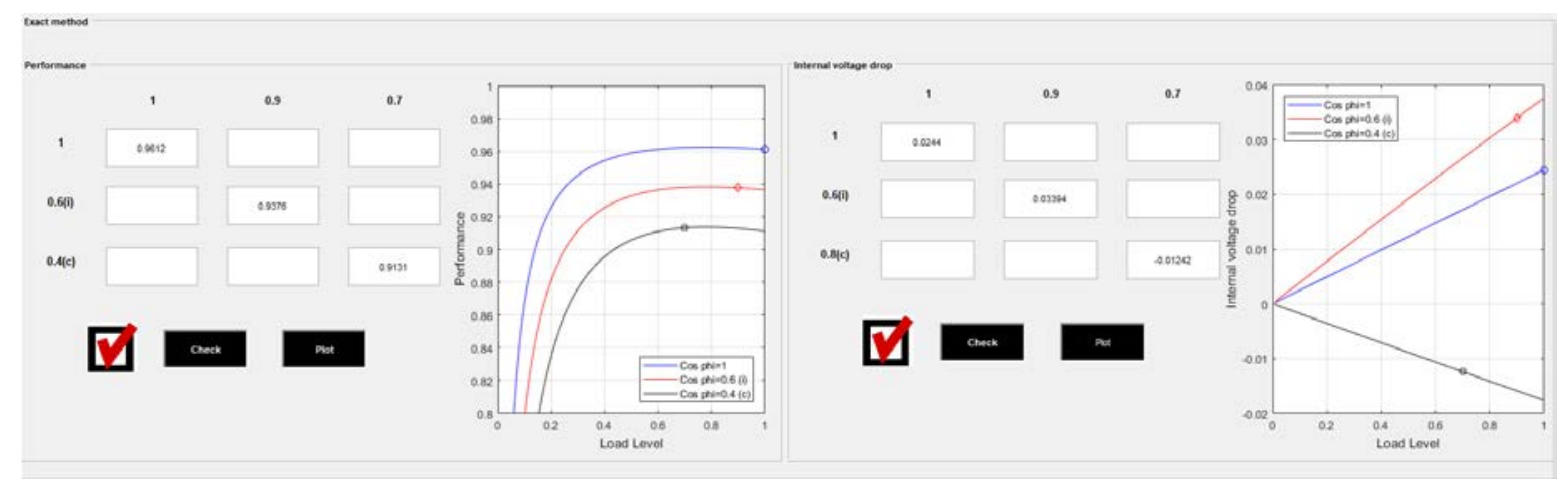

Figure 4. Checking the results of the second activity.

\subsubsection{Panel 3: Internal voltage drop and performance. Approximate method.}

During theoretical classes, the student is taught an alternative method for calculating performance and internal voltage drop. This is an approximate method, but in turn helps the student to relate concepts used during the sessions such as shortcircuit power, no-load power or load level. In this regard, a third panel has been designed where, by means of using the nine loads as in the previous panel, the student will calculate these two parameters following the following formulas:

$$
\eta \approx \frac{C \cdot S_{N} \cdot \cos \varphi_{2}}{C \cdot S_{N} \cdot \cos \varphi_{2}+P_{0}+C^{2} P_{C C}} \quad \varepsilon_{c} \approx C \cdot \varepsilon_{c c} \cdot \cos \left(\varphi_{c c}-\varphi_{2}\right)
$$

Thanks to the graphic representation of the exact performance and voltage drop curves, the student will be able to see how much big is the deviation introduced by the approximate method.

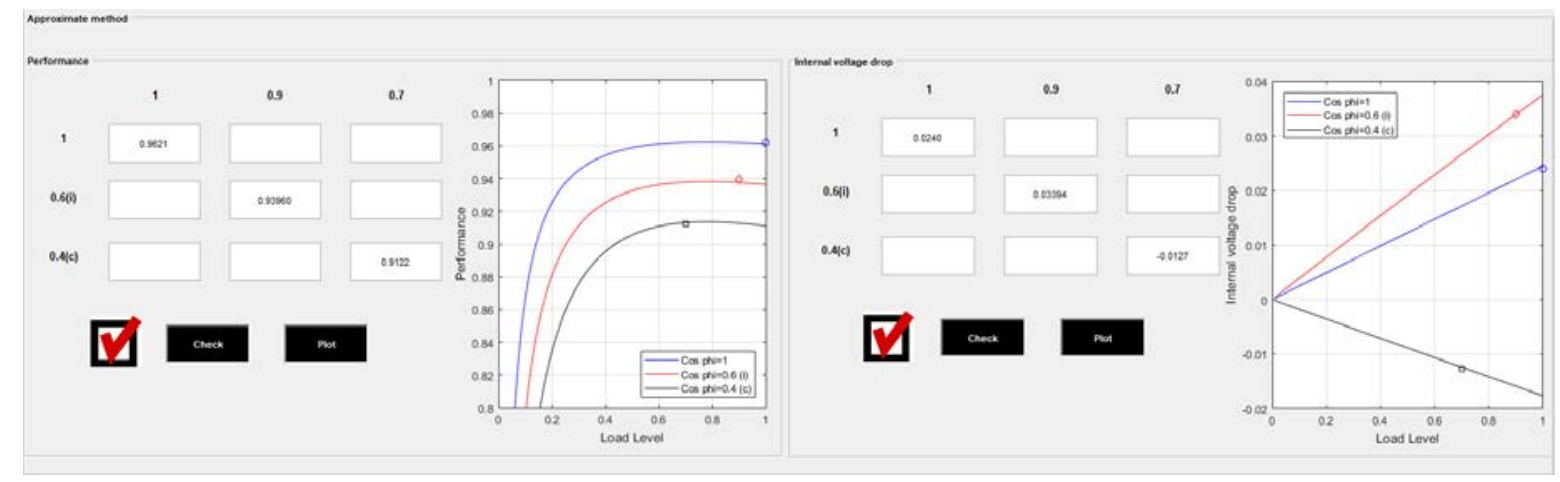

Figure 5. Checking the results of the third activity.

\subsection{Test description}

The test was designed to cover all the learning goals that the student was supposed to achieve during the practical session. It was formed by ten questions, structured as follows:

- 4 questions about the EEC parameters ( $\left.R_{F E}, R_{c c}, X_{\mu}, X_{C c}\right)$.

- 4 questions about performance and voltage drop ( 2 for exact and 2 for approximate method).

- 2 questions about conditions in the tests and physical meaning of the parameters.

Therefore, the student must answer a total of 10 questions. All data is given as screen captures of the virtual laboratory app, specially showing the resulting measures of the primary and secondary analysers. There are several versions of each question: every time the exam is called from the assessment platform, a version of each question is chosen randomly (as seen in Fig. 6). The questions are written in both Spanish and English. The aim of this system is to avoid repetition of the same exam for different students. If the solution is numerical, the right answer is considered with a certain tolerance. The last 2 questions are multiple choice ones, about conditions in the tests and physical meaning of the parameters (as seen in Fig. 7). In this case, each question has four possible answers. 


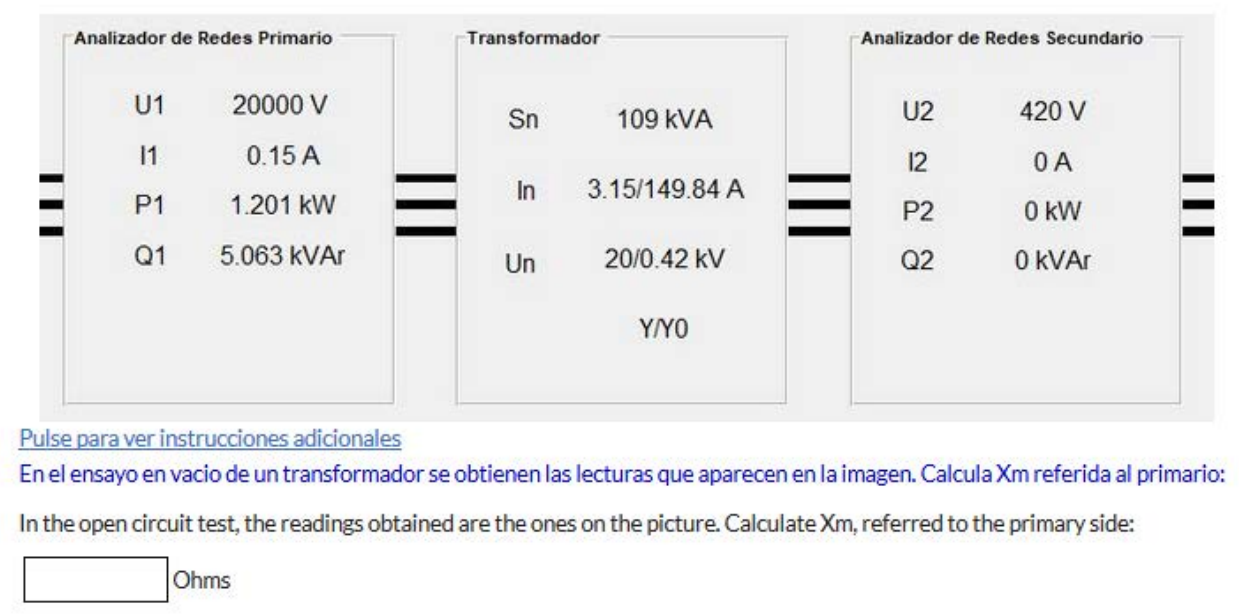

Figure 6. Example of question with numerical answer.

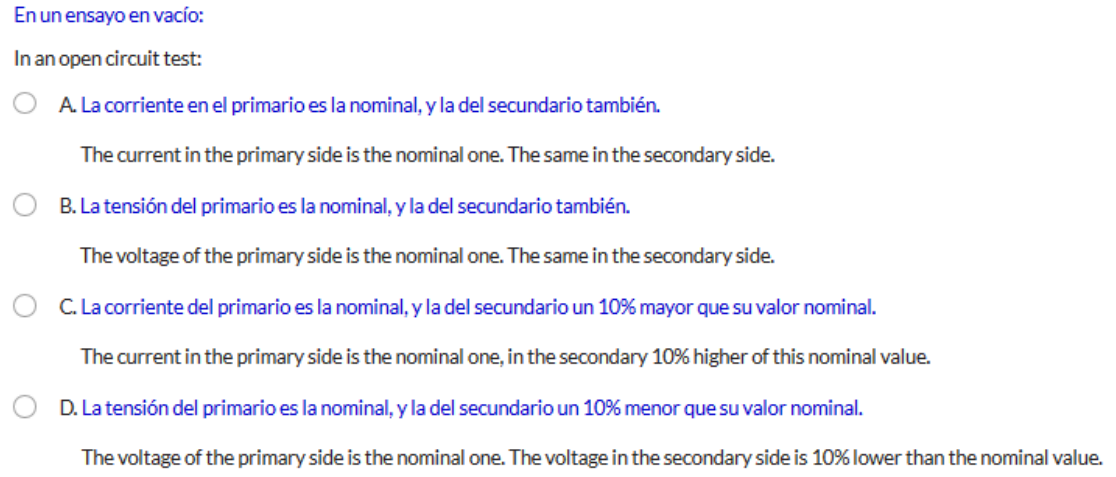

Figure 7. Example of multilingual multiple-choice question.

\subsection{Satisfaction survey description}

The survey was designed to cover the most important topics related to the objectives and the use of the app. It was formed by seven questions, where students had to give marks from one to five, one meaning fully disagree and five meaning totally agree. Those questions were:

1 Do you think that this application has been helpful when it comes to using electrical devices in the laboratory?

2 Do you think that this application has been helpful when it comes to the evaluation test?

3 Do you think that you have had enough time to complete all the activities that were requested in the application?

4 Do you think that similar applications should be used in the following lab lessons?

5 Do you find the graphic interface of the application easy to use?

6 Do you consider the application guide useful?

7 Do you think that this application has been helpful when it comes to understand all the concepts related to the subject?

\section{RESULTS}

\subsection{Assessment test}

This test has been used for assessment after practicing with the virtual lab during an hour and a half. The results of the test show very good outcome, especially for the first four questions dealing with parameters calculations: around $80-100 \%$ correct (Fig. 8). Another significant result is that questions 5 and 8 where mainly not answered. These questions deal with the approximate method of performance 
and voltage-drop calculation, which was the last part of the session, which nearly non student reached. In further sessions, the use of the application third panel, related to approximate method, will be removed due to insufficient time. Nonetheless, the rest of learning goals where completely achieved.

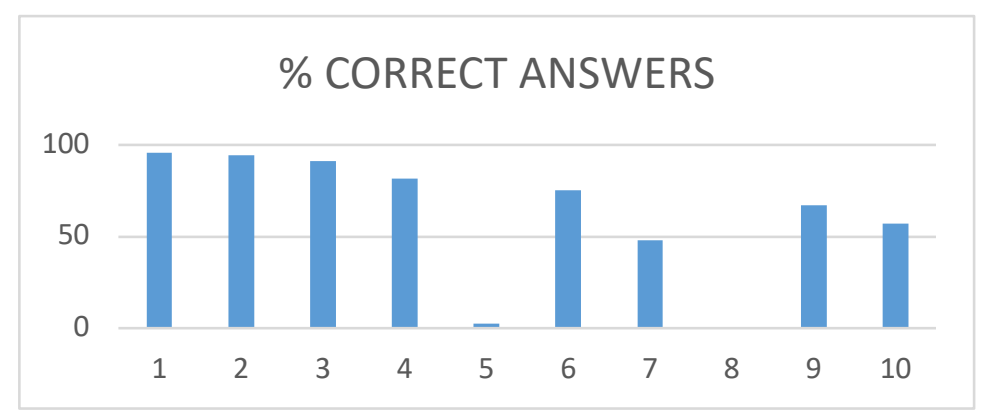

Figure 8. \% of correct answers for each question.

To establish if the students are making significant use of the virtual laboratory, and if it is helping students in their learning, a group of students were asked to take the test twice: pre-test, only with knowledge from theoretical sessions and the real laboratory, and post-test, after working with the virtual lab. The results (Fig. 9) show that the number of students who pass the test increases a $46 \%$ and the average mark improves from 4.4 to 7.69 over 10 . This shows how the application enhances learning.

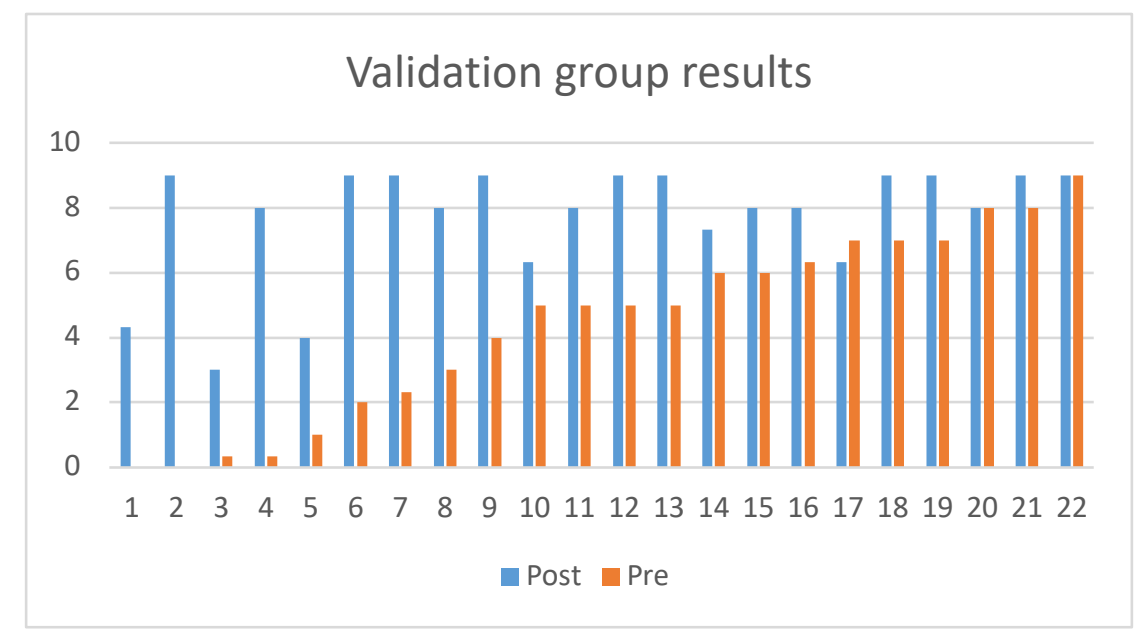

Figure 9. Comparison of the results before and after the app is used.

\subsection{Students satisfaction survey}

The survey has been used to obtain relevant information about the students opinion on the virtual laboratory here presented. Particularly, the survey asks for the performance of the application in their learning, at the level of theoretical concepts and procedural skills during the lab session, as well as to obtain information about whether the students have felt comfortable with the use of this type of application, and if they have had enough time to carry out the activities proposed in the virtual lab.

As can be seen in Fig. 10, the results obtained from the survey are good with an average score in the seven questions of 7.4. It is important to note that the best grades were obtained in those questions related to the understanding of concepts (7 and 2), procedural skills in the laboratory (1 and 2 ) and self-taught learning through the guide (6). Regarding the results of question 3 , many students found themselves short of time when they were doing the activities of the application (not laboratory) due to the large number of exercises that were requested, in the experience of teaching staff. As explained in the last subsection, panel three will be eliminated in the next academic year. Regarding question 5 , despite the fact that the grade is still very close to the average, some students encountered difficulties in the management of the program. 


\section{Average mark for each question}

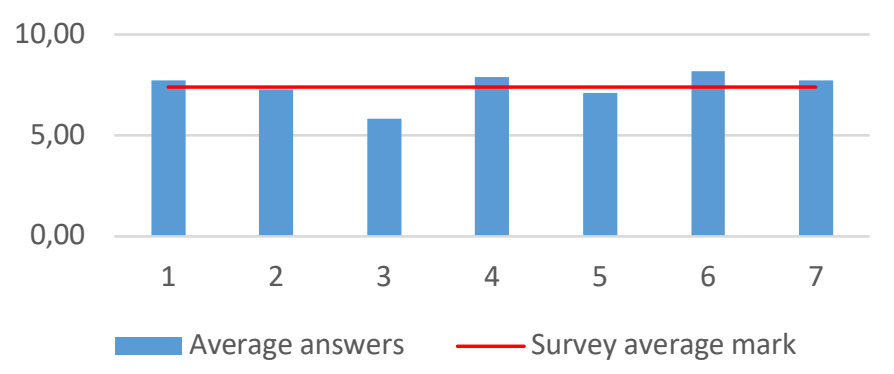

Figure 10. Average mark for each question in the satisfactory survey.

\section{CONCLUSIONS}

Based on the results of the study (test + survey), the authors conclude that there is a great benefit in using this type of applications specifically designed to obtain very concrete learning results. According to the perception and experience of the teaching staff, this is due to the fact that the application is fully adapted to the needs of the subject and therefore to the level of knowledge that the students need to acquire. Despite the enhancement of results by students (average score from 4.4 to 7.69 ) and the good impression they had on the application (average score 7.4), the following lines of improvement are proposed for new versions of the application:

- In view of the results of question three in the satisfaction survey, the authors conclude that a reduction in the number of proposed activities can be totally plausible, without detriment to the achievement of the learning objectives.

- Taking into account the results of question five of the satisfaction survey, even though the average of results is close to the general average and is the first version of the application and the first time it was put into operation at the university, the authors propose measures to improve fluency, the manageability of the program, as well as an increase in the margin of error in the check of results.

\section{ACKNOWLEDGEMENTS}

This project is supported by Universitat Politècnica de València through the Project of Innovation and Educational Improvement Program (PIME 2018-2019/B26).

\section{REFERENCES}

[1] Kozma, R.B., "The Implications of Cognitive Psychology for Compute-Based Learning Tools". Educational Technology, 27 (11), 20-25. 1987.

[2] Smit, C.P., Oosterhout, M., Wolff, P.F.J. R, Remedial Classroom teaching and computer-assisted learning with science students in Botswana. Int. J. Educational Development, 16 (2), 147-156. 1996.

[3] Cingi, C.C., "Computer aided education. $13^{\text {th }}$ International Educational Technology Conference. Procedia - Social and Behavioral Sciences, 103, 220-229.

[4] Şeker, S., "Computer - Aided Learning in Engineering Education. $2^{\text {nd }}$ World Conference on Educational Technology Researches - WCETR2012. Procedia - Social and Behavioral Sciences, 83, 739-742. 2013.

[5] Kollöffel, B. \& De Jong, T., "Conceptual Understanding of Electrical Circuits in Secondary Vocational Engineering Education: Combining Traditional Instruction with Inquiry Learning in a Virtual Lab. Journal of Engineering Education, 102(3), 375-393. 2013. 